\title{
A Review of Literature Pertaining to Head and Neck Squamous Cell Carcinoma with Emphasis on the Role of the Human Papilloma Virus
}

\author{
Samuel John Cartwright \\ Ear, Nose \& Throat Department, Royal Sussex County Hospital, Brighton, UK \\ Email: sjcartwright@doctors.org.uk \\ Received 18 July 2014; revised 15 August 2014; accepted 2 September 2014 \\ Copyright (C) 2014 by author and Scientific Research Publishing Inc. \\ This work is licensed under the Creative Commons Attribution International License (CC BY). \\ http://creativecommons.org/licenses/by/4.0/

(c) (i) Open Access

\section{Abstract}

Over the last decades the United Kingdom has seen a general increase in the incidence of head and neck squamous cell carcinoma (HNSCC). In addition to the classical risk factors of smoking and alcohol consumption there now appears that a viral aetiology is attributed to a significant proportion of HNSCC. A number of studies have indicated that human papillomavirus (HPV) infection is implicated with the subtype HPV 16 being found in many histopathological specimens. Further evidence suggests that HPV positive status is a prognostic factor related to a favourable outcome in head and neck cancers. This premise may have a significant impact on our approach to treating HNSCC. Patients belonging to stages I/II are traditionally treated with curative intent, single modality treatments that are either radiation or surgery alone. These treatment regimens are relatively aggressive and may lead to severe functional impairment. HPV patients may not require such an approach. This dissertation examines the current evidence which has given rise to these assertions, and explores the unresolved issues preventing HPV positive status becoming part of the management convention. The methodology involved a comprehensive literature review. The literature conclusions and limitations are discussed with reference to what is becoming accepted universally in this field and what remains to be elucidated. The different prognosis and treatment reaction to radiotherapy and chemotherapy by HPV positive tumours could be extrapolated to suggest that HPV status detection is required to plan treatment regimes. Indeed a separate subset of patients could potentially be categorised with an improvement in morbidity.

\section{Keywords}

Head \& Neck Cancer, Squamous Cell Cancer, HPV, Management 


\section{Introduction}

Head and neck cancer typically refers to squamous cell cancer which predominates in the oral cavity, oropharynx, hypopharynx, larynx, sinonasal tract and nasophaynx. It varies in grade from well-differentiated keratinising to undifferentiated non-keratinising.

It is the fifth leading cause of cancer worldwide with an estimated annual burden of around 560,000 incident cases and 301,000 deaths [1].

Trends in HNSCC have changed recently probably as a result of the impact of changes in tobacco smoking behaviour. There has been a reduction in head and neck cancers, such as those of the larynx in parallel with smoking habits [2]. However, certain subtypes have seen an increase in prevalence namely those of oropharyngeal and tonsillar cancer. These changes are attributable to a new risk factor in addition to the traditional ones; studies have shown this new entity to be human papilloma virus (HPV). The aim of this review is to explore the current knowledge and to examine the remaining controversies related to the role of HPV in HNSCC development and the potential ramifications.

The areas to be studied are:

- Diagnosis of HPV positive status in HNSCC.

- Prognostic significance of viral detection.

- Implications in the management and future directions.

The meta-analysis will attempt to review the background of knowledge relevant to HNSCC and how this has brought us to the current status quo. Each area will be addressed in turn with an explanation of what is known, and if apposite recent research will be used to illustrate where the contention in possible advancement remains. This is a domain where many questions persist; some papers on the subject lack objective evidence and have limitations of small sample size and insufficient statistical power. By examining these studies and any relevant meta-analyses, conclusions from both the quantitative and qualitative data can be drawn, thereby defining what currently vague is.

\section{Methods}

The Scottish Intercollegiate Guidelines Network (SIGN) for systematic literature reviews was used. Publications between the years 01/01/1970 to 01/02/2011 were located by means of PubMed, OmniMedicalSearch and Medline. They were manually searched for additional relevant non-Medline articles or abstracts. The total number of publications examined was 1675 .

Inclusion criteria were publications comparing HPV positive and negative groups, those examining mechanisms of HPV diagnosis and discussion of management of HNSCC. Papers examining the same subject matter were compared and the most recent one used in preference if the findings were similar. Molecular mechanisms in the development of HPV in HNSCC were collated from a number of review publications [3] [4].

Examination of papers pertaining to prognosis where examined on the basis of protocols defined by the Eastern Co-operative Oncology Group (ECOG) (Figure 1).

\begin{tabular}{|c|l|}
\hline Grade & ECOG \\
\hline 0 & Fully active, able to carry on all pre-disease performance without restriction \\
\hline 1 & $\begin{array}{l}\text { Restricted in physically strenuous activity but ambulatory and able to carry out work of a light or sedentary nature, } \\
\text { e.g., light house work, office work }\end{array}$ \\
\hline 2 & $\begin{array}{l}\text { Ambulatory and capable of all selfcare but unable to carry out any work activities. Up and about more than } 50 \% \text { of } \\
\text { waking hours }\end{array}$ \\
\hline 3 & Capable of only limited selfcare, confined to bed or chair more than $50 \%$ of waking hours \\
\hline 4 & Completely disabled. Cannot carry on any selfcare. Totally confined to bed or chair \\
\hline 5 & Dead
\end{tabular}

Figure 1. The criteria of ECOG on data analysis used on patient outcomes. 
This is a recognised tool used as a yardstick in measuring patient outcomes.

Data on molecular diagnostic tests was derived from general papers on the subject [5] and then specific papers on HPV detection. Certain conclusions in this dissertation were derived from constructing publications, particularly those by Kloazar [6], Kumar [7], Fakhry [8] and Weinberger [9].

Quantitative or qualitative methods are sufficient by themselves to capture a sufficient overview of the subject as it transcends many disciplines within this genre. This review therefore also utilised a multi-method triangulation of data approach.

The inherent limitations of the methodology are that it is based on reviewing existing literature and not individual patient data. An attempt to overcome these limitations by examination of the data which provided the most statistically significant information coupled with those of most relevance to this review has been made.

\section{Epidemiological Perspective}

Typically HNSCC presents late with advanced metastatic disease accounting for survival of $40 \%-50 \%$ at 5 years [10]. The most important risk factors identified are smoking and alcohol. Studies have suggested that these are synergistic in effect. McFarlane suggested a 50-fold increase in risk with combined smoking and alcohol consumption if heavy [11].

The effects of alcohol are not so clear as those of smoking. One possible action has been postulated to be by metabolism to acetaldehyde causing damage to DNA, this in-turn traps glutathione an important detoxification peptide in regard to carcinogens.

In addition, alcohol can also induce p450 enzyme CYP2E1, essential in the inactivation of a variety of procarcinogens found in alcoholic beverages and tobacco smoke. Carcinogens are also produced by tobacco smoke such as the polycyclic aromatic hydrocarbons (PAH) and the nicotine-derived nitrosamines. Recent studies have indicated that there is a strong coincidence of guanine to tyrosine transversion and sites of preferential formation of PAH adducts along the p53 gene. These mutations are seen in HNSCC [12].

A further factor implicated in HNSCC aetiology is diet and oral hygiene. Low consumption of fibre and vitamins have been linked to an increase in developing oro-pharyngeal cancer. The challenge remains to isolate these from high alcohol and tobacco consumption, this is compounded by the potential affect of deprived low-income demographics also having high consumption of these risk factors.

Now the the traditional risk factors are now being superseded by HPV. The American Society of Clinical Oncology [13] used data from the Surveillance Epidemiology and End Results (SEER) program registries to investigate the potential influence of HPV on incidence and of HNSCC in the United States (US) from 1973-2003.

One significant finding was the mean age at diagnosis was younger for HPV positive status in HNSCC than HPV negative (61.1 vs. 64.5 years; $\mathrm{P}<0.001$ ), and from 1973-2003, decreased significantly for HPV negative, but increased for HPV positive.

The proportion of HNSCC that is potentially HPV positive status was found to have increased in the US from 1973-2003, particularly among more recent birth cohorts.

It also showed that for HPV positive HNSCCs, age-adjusted incidence increased significantly from 19732003. The Annual Percent Change (APC) was 0.65. This was particularly evident among males (APC = 1.02), and whites (APC $=0.89)$.

The age bands show a definite change in younger ages, APCs for:

- 30 - 39 years $=1.46$

- 40 - 49 years $=1.92$

- 50 - 59 years $=0.61$

- $>60$ years $=-0.66$

These findings are echoed by that of the Oxford Cancer Intelligence Unit (OCIU) [14] which is a database for Head and Neck Cancer using the e-Atlas incorporating data from English, Scottish, Welsh and Northern Irish cancer registries and the Office for National Statistics. This data correlates with finding in the United States that a younger, male demographic is affected by HPV associated HNSCC.

\section{Relevant Anatomical Considerations \& Presentation}

\subsection{Classification}

The head and neck regions which are mainly affected by squamous cell carcinoma fall within the following: 
- The oral cavity, which includes the lips, buccal mucosa, anterior tongue, floor of the mouth, hard palate, and upper and lower gingiva.

- The pharynx, which includes the oropharynx, and hypopharynx. The oropharynx includes the tonsillar area, tongue base, soft palate, and the posterior pharyngeal wall; the hypopharynx includes the pyriform sinuses, the posterior surface of the larynx (postcricoid area), and the inferior, posterior, and lateral pharyngeal walls.

- The larynx, which is divided into three anatomic regions: the supraglottic region, the glottic larynx (true vocal cords and mucosa of the anterior and posterior commissures), and the subglottic larynx which extends to the inferior border of the cricoid cartilage.

\subsection{Clinical Presentation}

The clinical presentation of HNC varies with the primary site.

- The oral cavity usually presents as a painful/painless ulcer which fails to heal, a warty growth, halitosis and subsequently dysphagia.

- The pharynx:

-Oropharynx, around twenty percent of patients present with a neck lump as the only symptom. Sore throat, referred otalgia, odynophagia and muffled speech are common. Trismus is a late symptom and suggests pterygoid involvement.

-Hypopharynx, early symptoms include the sensation of a lump or discomfort in the throat. Latterly, the patient will present with progressive dysphagia. Hoarseness may occur as a result of invasion of the larynx or vocal cord paralysis. A patient with advanced disease may have anorexia and weight loss. There may be a history of food sticking and repeated aspirations leading to pneumonia.

- The larynx, clinical features are dictated by the primary tumour, secondary deposits and the general effects of the cancer. The symptoms and signs of laryngeal cancer depend on the way in which it is related to the upper aerodigestive tract. Hoarseness is the commonest and often the only presenting symptom. Dyspnoea and stridor are late symptoms and almost invariably indicate advanced tumour.

\section{Human Papilloma Virus as the New Entity}

A viral aetiology in HNSCC was first suggested in the 1980s when an association was made between morphological and histological lesions [15]. The incidence of head and neck cancers (HNC) has fallen overall in the United States in recent years, linked with reduced tobacco consumption. However, in contrast HPV associated cancer is rising, especially in young adults. The age adjusted incidence in tonsillar cancer increased 3.5-fold in women and 2.6-fold in men between 1970 and 2002 [16]. In the UK HNSCC of the tonsil in particular is increasing. In England there were 281 tonsillar cancers diagnosed in 1997; this has increased to 703 in 2007 [17].

The age of incidence is attributable to sexual behaviour and the number of lifetime partners both vaginal-sex and oral-sex. This complies with methods of HPV transmission and indeed HPV 16 seropositivity has been demonstrated to infer a 2-fold increased risk of developing oral cancer [18].

Establishing the role of HPV has been compounded by the heterogenicity of the tumours in addition to the multiple anatomical sites encompassed. There have been numerous publications studying HPV using various detection techniques. For example in situ hybridisation, Southern Blot hybridisation, Polymerase chain reaction; differing sampling methods such as biopsies, scrapes, oral rinses, brushes. This has lead to a variability in detection rates of $0 \%-100 \%$.

Non-standardisation of the multiple variables has failed to bring clarity to the arena.

One systematic meta-analysis performed by Kreimer [19] attempted to ascertain the prevalence of HPV positive status in HNSCC. Overall prevalence of HPV in HNSCC was estimated at 26\%. They analysed 60 eligible studies using PCR detection from 26 countries which included 5046 cases of squamous cell carcinomas; 2462 oral cancers, 969 oropharyngeal cancers and 1435 laryngeal cancers. HPV prevalence was $35.6 \%$ in oropharyngeal cancers, $23.5 \%$ in oral cancers and $24 \%$ in laryngeal cancers.

Of the subtypes of HPV, 16 was the commonest subtype. It was found in $86.7 \%$ of oropharyngeal, $68.2 \%$ of oral and $69.2 \%$ of laryngeal cancers. HPV 18 was the next most common but only found in $8 \%$ of oral, $3.9 \%$ of laryngeal and $1 \%$ of oropharyngeal cancers. Kreimers publication is currently most significant suggesting of HPV as a casual role in HNSCC. 


\section{Molecular Mechanisms}

HPVs are small deoxyribonucleic (DNA) viruses that infect various epithelial tissues including the epidermis (cutaneous types) and the epithelial linings of the upper respiratory system and anogenital tract (mucosotropic types). The difference in their ability to promote malignant transformation is the basis for the classification of HPVs into low and high-risk types.

This concept emanates from observations made more than 30 years ago [20] that some HPV types were more frequently found in cancers than in benign lesions, and was followed by a large number of studies that demonstrated that these risk categories reflect the inherent and differential abilities of the viruses to interfere with proliferation and stability of the genome of the infected cell.

A subset of mucosotropic HPVs that belong to the alpha genus, including the high-risk HPV types 16 and 18. These are associated with more than the majority of HPV in HNSCC. Much of the understanding on carcinogenesis is derived from studies in cervical carcinoma.

HPV 16 is the predominant form of HPV positive status in HNSCC. This has been quoted at $85 \%-95 \%$ which is higher than that of cervical cancers. It arises most commonly in the lingual and palatine tonsils [21] but is found in all anatomical categories of HNSCC.

Studies have shown the HPV subtypes linked with HNSCC are generally akin to that of cervical carcinoma. This possibly has implications with new cervical HPV vaccines, vide infra.

The effects of HPV are predominantly related to the E6 and E7 oncogenes (Figure 2). These bind and cause degradation of p53 and retinoblastoma tumour suppressor genes. The function of p53 is to arrest cells in growth phase 1 (G1) or induce apoptosis to allow host DNA to be repaired. E6 is of particular interest because it appears to have multiple roles in the cell and to interact with many other proteins. Its major role, however, is to mediate the degradation of p53, a major tumour protein, reducing the cell's ability to respond to DNA damage [22].

\section{Human Papilloma Virus Detection}

The association between HPV infection and cervical carcinoma is well established.

In HNSCC, however there is no direct equivalent to the transformation zone of the cervix in the oropharynx. Clinicians screening for HPV in gynaecology are fortunate because of a set screening process and treatment algorithm. A similar infrastructure dose not exist for HNSCC. There is evidence, however, that HPV is found predominantly in the crypts of the tonsils. This may constitute a parallel to the transformation zone.

Tumours from tonsillar crypts are not associated with dysplasia of surface epithelium; show lobular growth; are permeated by infiltrating lymphocytes; do not undergo clinically significant basaloid morphology [23].

Clinically HPV positive tumours present mostly at an early T stage and advanced nodal stage. In general, HPV associated oropharyngeal cancers at presentation are stage III or IV. Nodal metastasis is usually cystic and multi-level [24]. The viral load in HNSCC varies considerably. Available data suggests that oral cavity HPV viral load is lower than that of the cervix. From the studies analysed it is clear that a number of factors are responsible for this observation. Primarily the anatomical sites are not akin to that of the cervix and its transformation zone. Secondly the variable pattern of integration plays a role. Weinberger [9] demonstrated that HPV 16 viral load and p16 expression could be used to classify head and neck cancers into 3 profiles (Class I-HPV negative, p16 low; Class II—HPV positive, p16 low; Class III—HPV positive, p16 high). Class III tumours had a significantly increased 5 year survival.

Despite growing calls for routine HPV testing of all HNSCC the best method for HPV testing is not established. Both in situ hybridisation (Picture 1) and PCR are commonly used. For satisfactory classification in clinical practice HPV tests should reliably work on fixed cells and tissue. There is evidence that detection of high risk HPV by consensus polymerase chain reaction (PCR) alone is insufficient to accurately classify tumours. This is due to it providing many clinically irrelevant false positives. However, there is convincing evidence that the detection of p16 protein by immunohistochemistry can be used as a surrogate marker for the elaboration of oncogenic HPV proteins. Calls for standardisation of HPV testing in head and neck cancers have led to two diagnostic algorithms have emerging: the first advocates screening for p16 by immunohistochemistry followed by detection of HPV DNA by in situ hybridisation: the second recommends detection of p16 followed by consensus PCR.

A study by Robinson et al. [25] in 2010 took this a step further in an attempt to determine the most viable as 


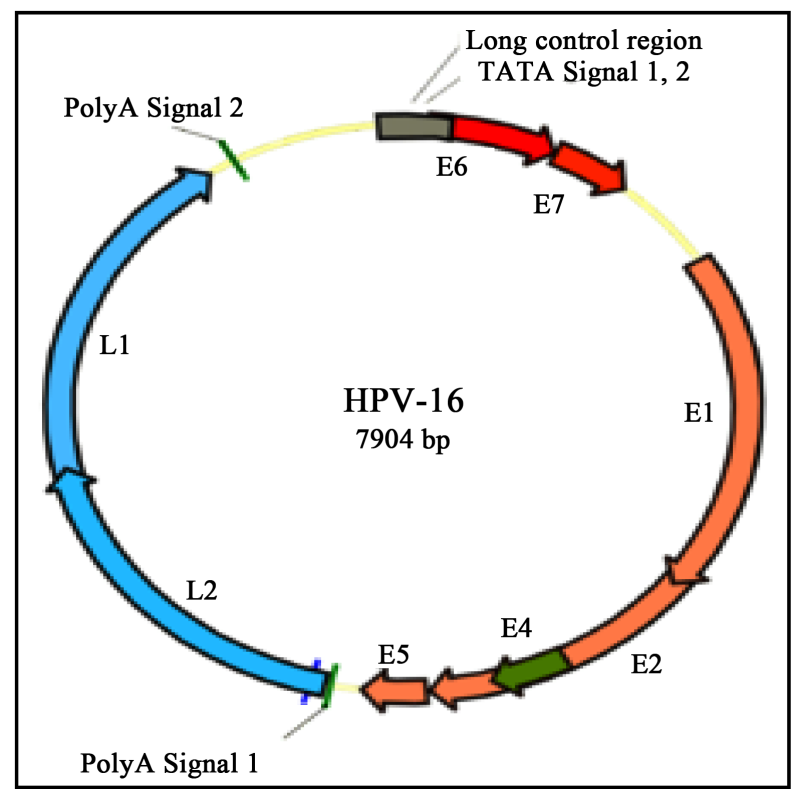

Figure 2. Integration of HPV into cell cycle. Together with E6, E7 serves to prevent apoptosis and promote cell cycle progression, thus priming the cell for replication of the viral DNA. E7 also participates in immortalisation of infected cells by activating cellular telomerase. Integration of HPV 16 correlates with a selective growth advantage and may allow the cancer cell to "outgrow" other cells.

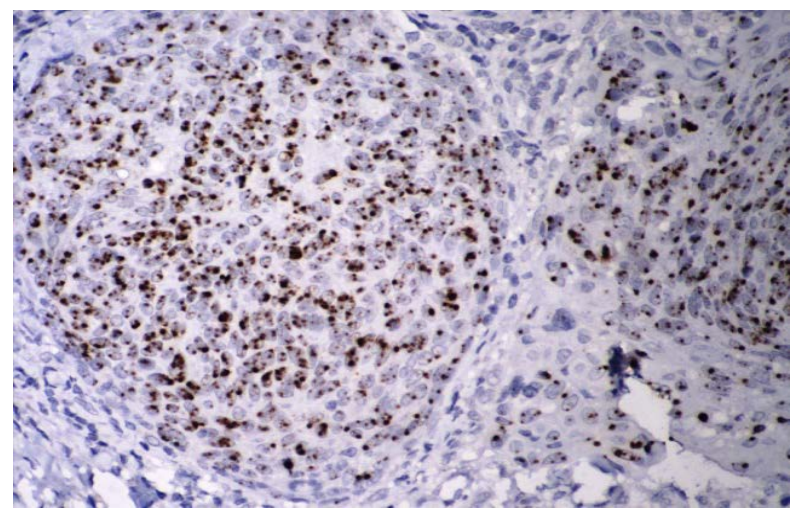

Picture 1. Showing HPV 16 positive in situ hybridisation.

say in HNSCC. They compared the polymerase chain reaction (PCR) assay, DNA-DNA in situ hybridization (ISH), and HPV 16 E7 PCR amplification in consecutively accessioned oropharyngeal cancers. 126 cases were tested by both PCR methods; 102 were positive by either for a maximum positive rate (MPR) of $81.0 \%$. Relative to the MPR, the sensitivities of PCR and E7 PCR were $90.2 \%$ and $72.5 \%$, respectively. Of 17 PCR+ cases tested by ISH, 14/14 unequivocally positive/negative were concordant. All cases (97/97) positive by either PCR assay were positive for p16. There was no relationship between level of histological differentiation and HPV status. ISH and PCR have comparable performance for the detection of HPV in oropharyngeal carcinomas. It was concluded that PCR is a suitable and economical assay that is comparable to ISH in sensitivity and may provide logistical advantages relative to ISH for assessing HPV status in oropharyngeal malignancies. However, it is imperative that appropriate sensitivity controls be in place for such assays.

The ultimate decider will be influence by sensitivity, specificity, reproducibility and cost. PCR methods require a high level of technical skill and are best used on fresh frozen samples. Mere detection of virus by non quantitative methods is unable to distinguish between transciptionally active (clinically relevant) from transcrip- 
tionally inactive (clinically irrelevant) HPV infections. This proposition in regard to globally accepted methods of detection is unfortunately not the only issue that seems to be preventing the advancement of HPV positive patients receiving alternative treatment regimens.

\section{Traditional Prognostic Factors in HNSCC}

\subsection{Site and TNM Stage}

The most important prognostic factors in HNSCC are site and TNM (tumour, node, metastasis) stage. Patients with tumours that are larger and have spread to nodes and other tissues have poorer survival. Guidelines for HNSCC from the Royal College of Pathologists state that other accepted features related to clinical outcome are grade, pattern of invasion, proximity of carcinoma to resection margins, and the presence of extranodal spread.

A large meta-analysis publication showed that extranodal spread more than halved the chances of surviving for five years (odds ratio 2.7, 95\% confidence interval 2.1 to 3.7 ) [26].

\subsection{Comorbid Illness}

The results of other large meta-analyses of clinical trial data illustrate that poor performance status that is poor fitness and the presence of comorbidities was associated with an adverse prognosis. One systematic review of the effect of comorbidity on survival from head and neck cancer (laryngeal) found that risk of death was significantly related to (hazard ratio 1.5 - 13.5, depending on the comorbidity).

Furthermore meta-analyses of clinical trial data showed that age is associated with a decreased survival [27]. This is relevant to the demographics of HNSCC as patients are generally considered to be younger (Table 1).

\section{Clinical Significance of HPV Positive Status in Head \& Neck Cancers}

The majority of studies suggest that HPV positive status is an important prognostic factor associated with a favourable outcome in HNSCC. The one recent prospective multi-centre clinical study is detailed below with a synopsis of the survival analysis which was the pertinent part of this study [28].

In survival analysis, the primary statistical end points were overall survival (death from all causes) and disease-specific survival (death from HNSCC). Event time distributions for these end points were estimated by use of the method of Kaplan and Meier (Figure 3) and compared by use of the log-rank statistic or the proportional hazards regression model. The assumption of proportional hazards for primary and recurrent cancers was not appropriate; therefore, survival models were stratified for this factor. The simultaneous effect of two or more factors was studied by use of stratified multivariate proportional hazards models. Factors tested for prognostic value included sex, age at diagnosis, race, tobacco and alcohol exposures, tumour stage, lymph node status, HPV presence, tumour location, and tumour grade.

Survival data were also compared separately for oropharyngeal and non-oropharyngeal HNSCC patients. there were three cancer deaths in 34 patients with HPV-positive and 10 cancer deaths in 26 patients with HPV negative oropharyngeal cancers. Disease-specific survival was significantly improved in the HPV positive oropharyngeal group (Hazard Ratio $(\mathrm{HR})=0.26 ; 95 \% \mathrm{CI}=0.07-0.98$; $\mathrm{P} \geq 0.05$ ). Among patients with nonoro-

Table 1. Concise summary of differences between HPV positive and HPV negative status in HNSCC.

\begin{tabular}{ccc}
\hline & HPV positive tumours & HPV negative tumours \\
\hline Anatomical site & Tonsil \& base of tongue & All sites \\
Histology & Non-keratinised & Keratinised \\
Age & Younger Cohorts & Older cohorts \\
Sex ratio & $3: 1$ men & $3: 1$ men \\
Stage & Tx, T1-2 & Variable \\
Risk factors & Sexual behaviour & Alcohol \& tabacco \\
Incidence & Increasing & Decreasing \\
Survival & Improved & Unchanging \\
\hline
\end{tabular}




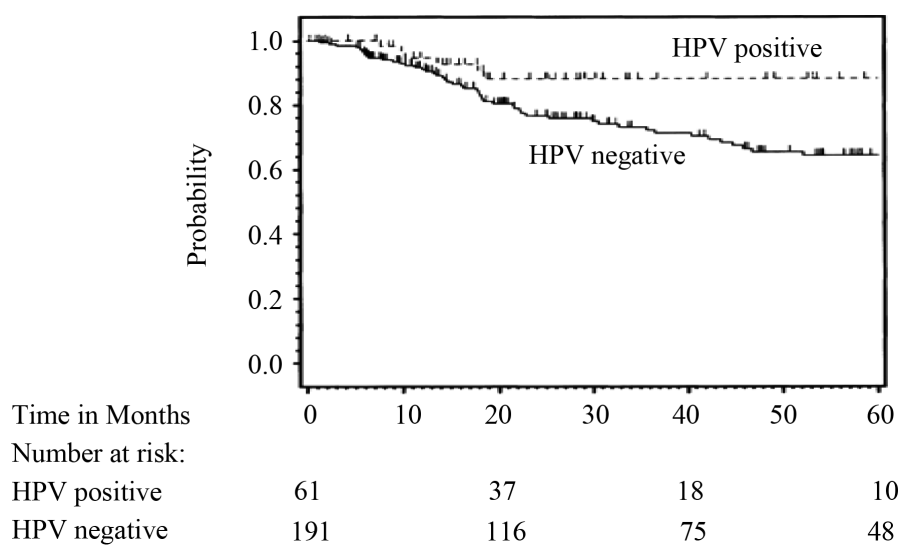

Figure 3. Kaplan-Meier plot of disease-specific survival for head and neck squamous cell carcinoma patients with human papillomavirus HPV)positive and HPV-negative tumors. Vertical ticks represent censored events. Patients with HPV-positive tumors had significantly improved disease-specific survival when compared with patients with HPV-negative tumors (log-rank, chi-squared $(1 \mathrm{df})=5.33$; $\mathrm{P}=0.02)$.

pharyngeal cancers, there were six cancer deaths in 28 patients with HPV-positive and 52 cancer deaths in 165 patients with HPV negative cancers. Disease-specific survival was similar in the two groups (HR $=0.62$; $95 \%$ $\mathrm{CI}=0.26-1.5 ; \mathrm{P}=0.28$ ).

A further paper which supports these findings is that of Fakhry [8]. After adjustment for age, tumour stage, and ECOG performance status, tumour HPV status was also statistically significantly associated with progression free survival (Figure 4). Patients with HPV positive tumours had a risk of progression that was $73 \%$ lower than that of patients with HPV negative tumours (adjusted HR $=0.27,95 \% \mathrm{CI}=0.10$ to $0.75, \mathrm{P} \geq 0.01$ ).

When the analysis was restricted to patients with oropharyngeal cancer, patients with HPV positive tumours had a $61 \%$ lower risk of death $(\mathrm{HR}=0.39,95 \% \mathrm{CI}=0.15$ to $1.05, \mathrm{P}=0.06)$ and a $62 \%$ lower risk of progression ( $\mathrm{HR}=0.38,95 \% \mathrm{CI}=0.12$ to $1.15, \mathrm{P}=0.09$ ) than patients with HPV negative tumours after adjustment for ECOG performance status.

One feature of Fakry's study was the separation of oropharyngeal cancer from the entir population of HNSCC. This allows greater interpretation in respect to these anatomical sub-categories. These studies also highlighted the difference in response to chemotherapy and radiotherapy regimens by HPV positive tumours meaning HPV status detection is required to individualise and plan treatment.

\section{Examining Current Treatment Options in HNSCC}

\subsection{Early Stage Disease}

In the region of $30 \%-40 \%$ of patients present with early stage I/II disease, curative intent is the premise of treatment using radiation or surgery in isolation. Both modalities result in similar rates of control and survival. The choice of treatment is usually therefore based on assessment of functional outcomes and other morbidities. If surgery is implemented primarily then adjuvant radiotherapy is added if positive or close margins exist in the resected specimen if there is evidence of bone erosion or pathological lymph nodes.

Early stage disease is associated with an excellent prognosis, however, these patients remain at high risk for recurrence/secondary tumours necessitating close monitoring.

\subsection{Resectable Locally Advanced Disease}

The dual considerations of maximising cure potential and maintaining functional status are fundamental to management in this scenario. Historically surgery is used in combination with postoperative radiotherapy.

\subsection{Definitive Radiotheraphy (RT)}

RT has been implemented as the primary treatment in all subsites of HNSCC and its role in comparison to sur 

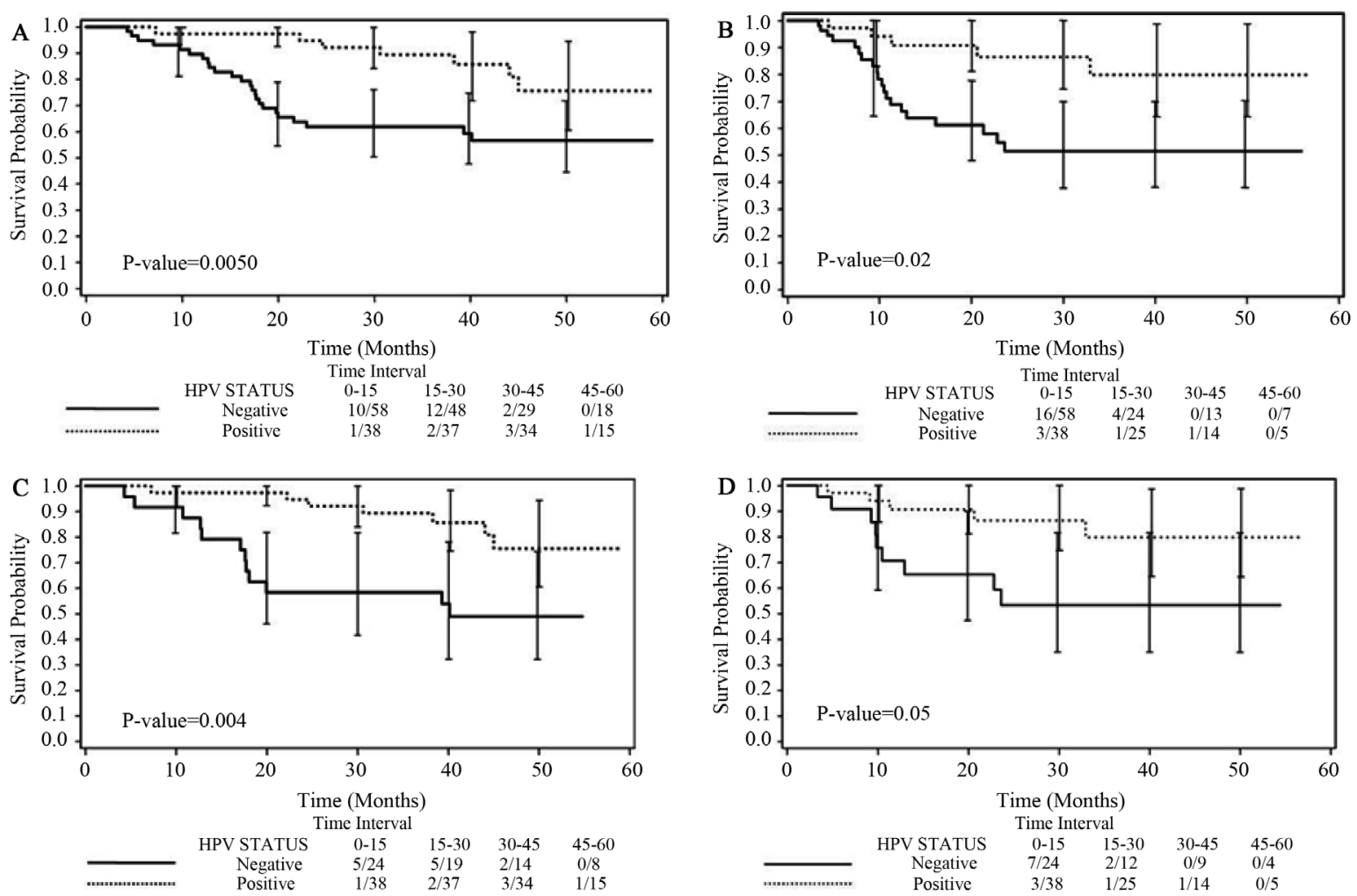

Figure 4. Kaplan-Meier curves for overall and progression-free survival stratified by tumor human papillomavirus (HPV) status. (A) Overall survival (OS) for the entire study population; (B) Progression-free survival (PFS) for the entire study population; (C) OS for patients with oropharynx cancer only; (D) PFS for patients with oropharynx cancer only. For all curves, 95\% confidence intervals for survival estimates at several time points are shown. Events per patients at risk are indicated for 15-month intervals.

gery has been continually evaluated [29].

RT as a single modality treatment remains an important option for early HNSCC and has produced high cure rates that are comparable to that of surgery. As a consequence the use of RT for early nodal disease was debated, as was the rational for elective irradiation in the clinically negative neck in patients at risk of occult metastasis [30]. Ordinarily, elective treatment of the head and neck corresponds to the chosen treatment of the primary tumour. RT is frequently used in early stage disease in oropharyngeal and hypopharyngeal carcinomas as the cure rate is akin to that achieved with surgical treatment but with lower morbidity [31].

\subsection{Postoperative Chemoradiotherapy}

Historically, the combination of RT and surgery for more advanced HNSCC was investigated in an effort to decrease recurrence rates in more advanced stage cancers for example those with gross organ destruction, bone/ cartilage invasion. The merits of surgery for eradicating bulky disease and RT for eliminating subclinical foci were a logical combination [32]. However, studies [33] reported a significant increase in toxicities, such as bone marrow suppression, mucositis and fibrosis. This lends reason to the premise that aggressive treatment should be reserved for patients with good performance status and high risk features e.g. extracapsular extension or positive surgical margins.

\subsection{Highly Conformational Radiation Therapy}

Intensity-modulated radiotherapy (IMRT) is a technology designed to increase the conformity of the radiation dose thereby reducing the toxicity in normal tissues. Computerised Tomography (CT) is used to design volumetric portals and the resulting radiation dose distribution [34]. Inverse planning is used in IMRT to elucidate target doses for the tumour and surrounding tissue. IMRT uses a large number of radiation portals to achieve the 
tailored distribution. Excellent loco-regional control has been demonstrated in current data from single institutions in oropharyngeal cancer [35].

\subsection{Concurrent Chemotherapy and Radiation Therapy}

Combined surgery, RT and chemotherapy is critical to curative management of locally advanced HNSCC. Chemotherapy improves the efficacy through its radiosensitising ability in loco-regional disease and also providing systemic therapy against metastatic disease. Concurrent regimens are standard treatment in the setting of locally advanced disease and high risk pathology. This reflects the development of a multidisciplinary approach to HNSCC.

Therapies targeting Epidermal Growth Factor Receptor (EGFR) EGFR is commonly over-expressed in HNSCC. It is a member of the ErbB growth factor receptor kinase therapy. Its over-expression has been associated with disease recurrence and decreased survival. Several strategies have been developed to target EGFR. Cetuximab, an immunoglobulin G1 chimeric monoclonal antibody directed against EGFR, is the first molecularly targeted agent to improve survival in patients with HNSCC [36].

One recent study, conducted at Georgetown Lombardi Comprehensive Cancer Center, analysed tumours from sequentially treated patients between 2007 and 2009 with HNSCC that were treated with cetuximab and intensity-modulated radiation therapy (IMRT). Out of twenty patients, six had stage III disease and fourteen had stage IVa disease. The median age was 63 (age ranged from 31 to 78). The patients received IMRT for seven to eight weeks (median dose was $72.0 \mathrm{~Gy}$ ). Cetuximab was given for a median of seven cycles. Primary tumour sites included oral cavity (1), oropharynx (12), hypopharynx (1), larynx (5), and with one site unknown. Oneyear progression free survival rates were 100 percent for those with HPV-positive tumors and 31 percent for those who were HPV negative. Two year progression free survival rates were 60 percent for those with HPV compared to 23 percent for those with HPV negative tumours $(P=0.05)$ [37].

This is a relatively low powered study but represents the current level of research. It is one of a number of emerging studies which should eventually progress to separating the treatment arms of those with/without HPV positive status and giving reduced duration/cycles of IMRT.

\section{Vaccination}

HPV vaccines for example GARDASIL ${ }^{\circledR}$ is a quadriavalent vaccine that protects against HPV types $6,11,16$ and 18. It is a killed, inactivated vaccine. It may provide some protective benefit as a preventative as it is effective against the main protagonist HPV sub type 16, although there are other sub types linked with HNSCC.

In October 2009, CERVARIX ${ }^{\circledR}$ was approved in females aged $10-25$. This is a bivalent vaccine that protects against HPV 16 and 18. It is a denatured inactivated vaccine [38]. There are ongoing trials involving the use of HPV vaccines to treat patients with advanced or recurrent HPV associated cancers, but currently there is no published data showing to the effectiveness of these immunotherapy based treatments.

There is a postulated protective affect from vaccination, although it is premature to suggest this definitively, their will be a significant lag time before this is show in epidemiological data.

It is difficult to estimate the impact of global vaccination against HPV associated cancers.

It will be interesting to see if this is borne out in the future and changes in ratios between males and females increase as a result. It is conceivable that extending the vaccination to men may occur, but this is likely to be based on its cost effectiveness.

\section{Summary \& Conclusion}

This review has set out to define/identify that a difference exists between HPV positive HNSCC patients and that identification of HPV status is key. Several papers purport a biological distinction existing between HPV positive and negative patients; this is likely a result of the pathogenesis of cancer by HPV. One point that is not clearly defined is the molecular response to treatment to account for this difference. Indeed no such studies have broached this subject and all assertions in the literature rely on the statistical differences to account for this rather than firmly elucidated mechanisms.

In drawing together knowledge on HPV positive status in HNSCC, additional salient questions are raised. The data incorporated in this review clearly suggests a distinction between the HPV positive and negative groups, 
but is this enough in itself to warrant changing the regimens within this subset of patients and observing the effects? In the absence of any alternative data, should the reality of reduced chemoradiotherapy exposure for HPV positive patients be the next logical step [39]? The potential benefits improved morbidity without worsening mortality.

Much of this stems from the lack of homogenicity of HNSCC, and the multiple anatomical sites encountered throughout the aerodigestive tract. This point cannot be overemphasised as it has resulted in difficulty in direct comparison of findings [40]. Unfortunately, the majority of the available literature currently does not sub-categorise these groups and refers to HNSCC as one entity. The difficulty has been to establish firm conclusions which are not superficial in regard to the data on which their supposition is based. Evidence suggests that there is a greater susceptibility of oropharyngeal and tonsillar cancer to HPV infection, however, several studies have grouped together HNSCC and have failed to specify the origin of the tumours or the site specific prevalence of HPV.

Coupled with this there has been a lack of progression from the theoretical concepts into actual clinical practice. Progression has therefore stalled, and although research is ongoing, none of the major groups have released data on new initiatives. This is frustrating, as it has a direct impact on the morbidity of the HPV positive patient group.

Nonetheless, HPV as a definitive risk for HNSCC is now established affecting those of younger age, male gender, non-smoking and non-drinking status. Furthermore, HPV positive status independent of these has been demonstrated as being an indicator in overall survival of HNSCC.

It is evident that a multitude of questions remain to be answered and issues to be addressed. Lack of definitive answers exists for some of the key questions proposed to be investigated in this review. Indeed additional questions other than those originally noted have come to light. These include: should site specific HNSCC be studied in isolation? Should trials with variable dose radiation be commenced with HPV positive tumours in separate treatment groups determining effect on cure in relation to morbidity?

Identification of the best tests is needed for HPV diagnosis whether that is PCR or a combination of hybridisation. The limitations of any single detection assay have been shown to be offset using algorithms that combine the strength of complementary assays.

Primary and secondary prevention also remains an area to be explored, including use of HPV vaccines against infection and therapeutic vaccines for loco-regional recurrence.

Despite this area receiving much more attention of late, research which will eventually allow advancement into the clinical domain is still very much preliminary. Research that does exist has highlighted the many issues in prospectively evaluating response to treatment and if this represents a true difference in natural history between HPV positive and negative groups in the absence of any therapy.

This seems to be the direction of research (as was noted from the outset), but no data from any ongoing trials has been released.

In conclusion, the challenge remains of designing clinical trials which utilise the "best" means of HPV detection, allowing stratification of these groups leading to the identification of novel modes of treatment. The proviso to any new regimen is retaining the chance of cure whilst reducing the co-morbidities.

Assimilation of data remains a problem as groups studying this field have adhered to their own individual designs and therefore no universal platform exists from which to readily extrapolate into a treatment algorithm. Only well designed studies will determine what promised to be an area of great potential is eventually realised.

One concern is that without sound scientific principles addressing all the questions originally raised in this review compounding factors will affect results and further slow progress. Researchers will be concerned about how to progress without causing decreased survival at the expensive of short term quality of life goals as well as the ethical dilemma. The subject now resides at a crossroad, it requires a study to carry the mantle beyond what is currently known [41] [42].

\section{References}

[1] Jemal, A., Seigel, R., Ward, E., et al. (2007) Cancer Statistics. CA: A Cancer Journal for Clinicians, 57, 43-66. http://dx.doi.org/10.3322/canjclin.57.1.43

[2] Sturgis, E.M., Wei, Q. and Spitz, M.R. (2004) Descriptive Epidemiology and Risk Factors for Head and Neck Cancer. Seminars in Oncology, 31, 726-733. http://dx.doi.org/10.1053/j.seminoncol.2004.09.013 
[3] Munger, K. and Howley, P.M. (2002) Human Papilloma Virus Immortalization and Transformation Functions. Virus Research, 89, 213-218. http://dx.doi.org/10.1016/S0168-1702(02)00190-9

[4] Hobbs, C.G., Sterne, J.A.C., Bailey, M., Heyderman, R.S., Birchall1, M.A. and Thomas, S.J. (2006) Human Papilloma Virus and Head and Neck Cancer: A Systematic Review and Meta-Analysis. Clinical Otolaryngology, 31, 259-266. http://dx.doi.org/10.1111/j.1749-4486.2006.01246.x

[5] Palka, K.T., Slebos, R.J. and Chungemail, C.H. (2008) Update on Molecular Diagnostic Tests in Head and Neck Cancer. Seminars in Oncology, 35, 198-210. http://dx.doi.org/10.1053/j.seminoncol.2008.03.002

[6] Oken, M.M., Creech, R.H., Tormey, D.C., Horton, J., Davis, T.E., McFadden, E.T. and Carbone, P.P. (1982) Toxicity and Response Criteria of the Eastern Cooperative Oncology Group. American Journal of Clinical Oncology, 5, 649655. http://dx.doi.org/10.1097/00000421-198212000-00014

[7] Klozar, J., Kratochvil, V., Salakova, M., Smahelova, J., Vesela, E., Hamsikova, E., Betka, J. and Tachezy, R. (2008) HPV Status and Regional Metastasis in the Prognosis of Oral and Oropharyngeal Cancer. European Archives of OtoRhino-Laryngology, 265, S75-S82. http://dx.doi.org/10.1007/s00405-007-0557-9

[8] Kumar, B., Cordell, K.G., Lee, J.S., Prince, M.E., et al. (2007) Response to Therapy Outcomes in Oropharyngeal Cancer Are Associated with Biomarkers Including Human Papilloma Virus, Epidermal Growth Factor, Gender and Smoking. International Journal of Radiation Oncology*Biology*Physics, 69, S109-S111. http://dx.doi.org/10.1016/j.ijrobp.2007.05.072

[9] Fakhry, C., Westra, W.H., Li, S.G., Cmelak, A., Ridge, J.A., Pinto, H., Forastiere, A. and Gillison, M.L. (2008) Improved Survival of Patients with Human Papilloma Positive Head and Neck Squamous Cell Carcinoma in a Prospective Clinical Trial. Journal of the National Cancer Institute, 100, 261-269. http://dx.doi.org/10.1093/jnci/djn011

[10] Weinberger, P.M., Yu, Z.W., Haffty, B.G., Kowalski, D., Harigopal, M., Brandsma, J., Sasaki, C., Joe, J., Camp, R.L., Rimm, D.L. and Psyrri, A. (2006) Molecular Classification Identifies a Subset of Human Papilloma Virus Assocaited Oropharyngeal Cancers with Favourable Prognosis. Journal of Clinical Oncology, 24, 736-747. http://dx.doi.org/10.1200/JCO.2004.00.3335

[11] Oken, M.M., Creech, R.H., Tormey, D.C., Horton, J., Davis, T.E., McFadden, E.T. and Carbone, P (1982) Toxicity and Response Criteria of the Eastern Cooperative Oncology Group. American Journal of Clinical Oncology, 5, 649-655. http://dx.doi.org/10.1097/00000421-198212000-00014

[12] American Society of Clinical Oncology. www.asco.org

[13] Carvalho, A.L., Nishimoto, I.N., Califano, J.A. and Kowalski, L.P. (2005) Trends in Incidence and Prognosis for Head and Neck Cancer in the United States: A Site-Specific Analysis of the SEER Database. International Journal of Cancer, 114, 806-808. http://dx.doi.org/10.1002/ijc.20740

[14] Oxford Cancer Intelligence Unit (OCIU). http://www.ociu.nhs.uk

[15] Mignogna, M.D., Fedele, S. and Russo, L.L. (2004) The World Cancer Report and the Burden of Oral Cancer. European Journal of Cancer Prevention, 13, 139-142. http://dx.doi.org/10.1097/00008469-200404000-00008

[16] Macfarlane, G.J., Zheng, T., Marshall, J.R., Boffetta, P., Niu, S., Brasure, J., Merletti, F. and Boyle, P. (1995) Alcohol, Tobacco, Diet and the Risk of Oral Cancer: A Pooled Analysis of Three Case-Controlled Studies. European Journal of Cancer Part B: Oral Oncology, 318, 181-187. http://dx.doi.org/10.1016/0964-1955(95)00005-3

[17] Brennan, J.A., Boyle, J.O., Koch, W.M., Goodman, S.N., Hruban, R.H., Eby, Y.J., Couch, M.J., Forastiere, A.A. and Sidransky, D. (1995) Association between Cigarette Smoking and Mutation of the p53 Gene in Squamous-Cell Carcinoma of the Head and Neck. The New England Journal of Medicine, 332, 712-717. http://dx.doi.org/10.1056/NEJM199503163321104

[18] Syrjänen, K., Syrjänen, S., Lamberg, M., Pyrhönen, S. and Nuutinen, J. (1983) Morphological and Immunohistochemical Evidence Suggesting Human Papilloma Virus (HPV) Involvement in Oral Squamous Cell Carcinogenesis. International Journal of Oral Surgery, 12, 418-424. http://dx.doi.org/10.1016/S0300-9785(83)80033-7

[19] Kreimer, A.R., Clifford, G.M., Boyle, P. and Franceschi, S. (2005) Human Papilloma Virus Types in Head and Neck Squamous Cell Carcimomas Worldwide: A Systematic Review. Cancer Epidemiology, Biomarkers \& Prevention, 14, 467-475. http://dx.doi.org/10.1158/1055-9965.EPI-04-0551

[20] René Leemans, C., Braakhuis, B.J.M. and Brakenhoff, R.H. (2011) The Molecular Biology of Head and Neck Cancer: HPV-Infected HNSCC. Nature Reviews Cancer, 11, 9-22. http://dx.doi.org/10.1038/nrc2982

[21] Hammarstedt, L., Lindquist, D., Dahlstrand, H., Romanitan, M., Dahlgren, L.O., Joneberg, J., Creson, N., Lindholm, J., Ye, W., Dalianis, T. and Munck-Wikland, E. (2006) Human Papillomavirus as a Risk Factor in the Incidence of Tonsillar Cancer. International Journal of Cancer, 119, 2620-2623. http://dx.doi.org/10.1002/ijc.22177

[22] Termine, N., Panzarella, V., Falaschini, S., Russo, A., Matranga, D., Lo Muzio, L. and Campisi, G. (2008) HPV in Oral Squamous Cell Carinoma vs Head and Neck Squamous Cell Carcinoma Biopsies: A Meta-Analysis (1988-2007). Annals of Oncology, 19, 1681-1690. http://dx.doi.org/10.1093/annonc/mdn372 
[23] Klussmann, J.P., Weissenborn, S.J., Wieland, U., Dries, V., Kolligs, J., Jungehuelsing, M., Eckel, H.E., Dienes, H.P., Pfister, H.J. and Fuchs, P.G. (2011) Prevalence Distribution and Viral Load of Human Papilloma Virus 16 DNA in Tonsillar Carcinomas. Cancer, 92, 2875-2884. http://dx.doi.org/10.1002/1097-0142(20011201)92:11<2875::AID-CNCR10130>3.0.CO;2-7

[24] Begum, S. and Westra, W.H. (2008) Basaloid Squamous Cell Carcinoma of the Head and Neck Is a Mixed Variant That Can be Further Resolved with HPV Status. The American Journal of Surgical Pathology, 32, 1044-1050. http://dx.doi.org/10.1097/PAS.0b013e31816380ec

[25] Agoston, E.S., Robinson, S.J., Mehra, K.K., Birch, C., Semmel, D., Mirkovic, J., Haddad, R.I., Posner, M.R., Kindelberger, D., Krane, J.F., Brodsky, J. and Crum, C.P. (2010) Polymerase Chain Reaction Detection of HPV in Squamous Carcinoma of the Oropharynx. American Journal of Clinical Pathology, 134, 36-41. http://dx.doi.org/10.1309/AJCP1AAWXE5JJCLZ

[26] Burtness, B., Goldwasser, M.A., Flood, W., Mattar, B., Forastiere, A.A. and Eastern Cooperative Oncology Group (2005) Phase III Randomized Trial of Cisplatin plus Placebo Compared with Cisplatin plus Cetuximab in Metastatic/Recurrent Head and Neck Cancer: An Eastern Cooperative Oncology Group Study. Journal of Clinical Oncology, 23, 8646-8654. http://dx.doi.org/10.1200/JCO.2005.02.4646

[27] Royal College of Pathologists (2005) Standards and Datasets for Reporting Cancers: Datasets for Histopathology Reports on Head and Neck Carcinomas and Salivary Neoplasms. 2nd Edition. http://www.rcpath.org/Resources/RCPath/Migrated\%20Resources/Documents/G/G112_NeckDissectionDataset_Nov1 3.pdf

[28] Bourhis, J., Overgaard, J., Audry, H., Ang, K.K., Saunders, M., Bernier, J., et al. (2006) Hyperfractionated or Accelerated Radiotherapy in Head and Neck Cancer: A Meta-Analysis. The Lancet, 368, 843-854. http://dx.doi.org/10.1016/S0140-6736(06)69121-6

[29] Gillison, M.L., Koch, W.M., Capone, R.B., Spafford, M., Westra, W.H., Wu, L., Zahurak, M.L., Daniel, R.W., Viglione, M., Symer, D.E., Shah, K.V. and Sidransky, D. (2000) Evidence for a Causal Association between Human Papilloma Virus and Head and Neck Cancers. Journal of the National Cancer Institute, 92, 709-720. http://dx.doi.org/10.1093/jnci/92.9.709

[30] Mead, P.H. (2004) Surgery or Radiotherapy for Tonsil Cancer? Cancer, 16, 195-198. http://dx.doi.org/10.1002/1097-0142(196302)16:2<195::AID-CNCR2820160208>3.0.CO;2-7

[31] Jesse, R.H. and Fletcher, G.H. (1977) Treatment of the Neck in Patients with Squamous Cell Carcinoma of the Head and Neck. Cancer, 39, 868-872. http://dx.doi.org/10.1002/1097-0142(197702)39:2+<868::AID-CNCR2820390724>3.0.CO;2-N

[32] Mendenhall, W.M., Morris, C.G., Amdur, R.J., Hinerman, R.W., Malyapa, R.S., Werning, J.W., Lansford, C.D. and Villaret, D.B. (2006) Definative Radiotherapy for Tonsillar Squamous Cell Carcinoma. American Journal of Clinical Oncology, 29, 290-297. http://dx.doi.org/10.1097/01.coc.0000209510.19360.f9

[33] Vikram, B. and Farr, H.W. (1983) Adjuvant Radiation Therapy in Locally Advanced Head and Neck Cancer. CA: A Cancer Journal for Clinicians, 33, 134-138. http://dx.doi.org/10.3322/canjclin.33.3.134

[34] McCubbin, M., et al. (2010) Head and Neck Squamous Cell Carcinoma and Human Papillomavirus. Seton Network Notes, 10.

[35] Cooper, J.S., Pajak, T.F., Forastiere, A.A., Jacobs, J., Campbell, B.H., Saxman, S.B., Kish, J.A., Kim, H.E., Cmelak, A.J., Rotman, M., Machtay, M., Ensley, J.F., Chao, K.S., Schultz, C.J., Lee, N., Fu, K.K. and Radiation Therapy Oncology Group 9501/Intergroup (2004) Post Operative Concurrent Radiotherapy and Chemotherapy for High Risk Squamous Cell Carcinoma of the Head and Neck. The New England Journal of Medicine, 350, 1937-1944.

[36] Lee, N., Puri, D.R., Blanco, A.I. and Chao, K.S.C. (2007) Intensity-Modulated Radiation Therapy in Head and Neck Cancers: An update. Head and Neck, 29, 387-400. http://dx.doi.org/10.1002/hed.20332

[37] Bonner, J.A., Harari, P.M., Giralt, J., et al. (2006) Radiotherapy plus Cetuximab for Squamous-Cell Carcinoma of the Head and Neck. The New England Journal of Medicine, 354, 567-578. http://dx.doi.org/10.1056/NEJMoa053422

[38] Georgetown University Medical Center (2010) Improved Outcomes for HPV-Positive Head and Neck Cancer with Cetuximab and IMRT, Study Suggests. http://www.sciencedaily.com/releases/2010/10/101025133743.htm

[39] Goldenberg, D., Begum, S., Westra, W.H., Khan, Z., Sciubba, J., Pai, S.I., Califano, J.A., Tufano, R.P. and Koch, W.M. (2008) Cystic Lymph Node Metastasis in Patients with Head and Neck Cancer: An HPV-Associated Phenomenon. Head and Neck, 30, 898-903.

[40] Pai, S.I. and Westra, W.H. (2009) Molecular Pathology of Head and Neck Cancer: Implications for Diagnosis, Prognosis and Treatment. Annual Review of Pathology, 4, 49-70.

http://dx.doi.org/10.1146/annurev.pathol.4.110807.092158

[41] Al-Bakkal, G., Ficarra, G., McNeill, K., Eversole, L.R., Sterrantino, G. and Birek, C. (1999) Human Papilloma Virus 
Type 16 E6 Gene Expression in Oral Exophytic Epithelial Lesions as Detected in Situ rtPCR. Oral Surgery, Oral Medicine, Oral Pathology, Oral Radiology and Endodontics, 87, 197-208.

http://dx.doi.org/10.1016/S1079-2104(99)70273-8

[42] The HPV Connection (2010). http://www.oralcancerfoundation.org/hpv/ 
Scientific Research Publishing (SCIRP) is one of the largest Open Access journal publishers. It is currently publishing more than 200 open access, online, peer-reviewed journals covering a wide range of academic disciplines. SCIRP serves the worldwide academic communities and contributes to the progress and application of science with its publication.

Other selected journals from SCIRP are listed as below. Submit your manuscript to us via either submit@scirp.org or Online Submission Portal.
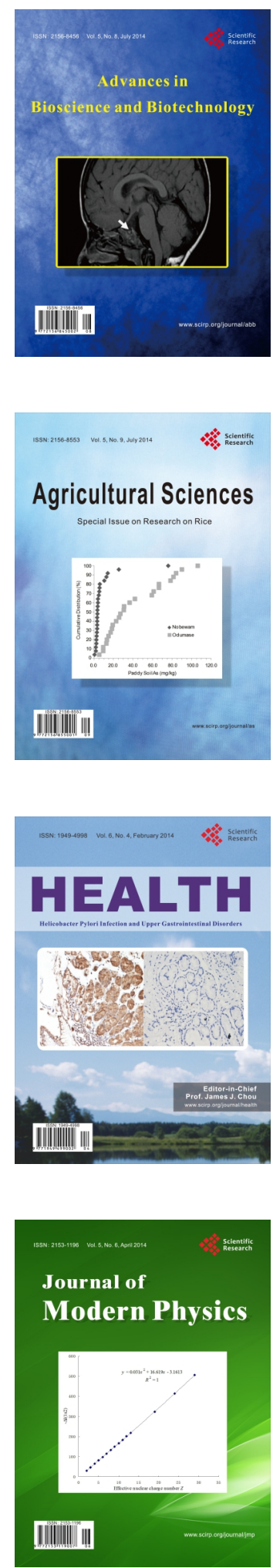
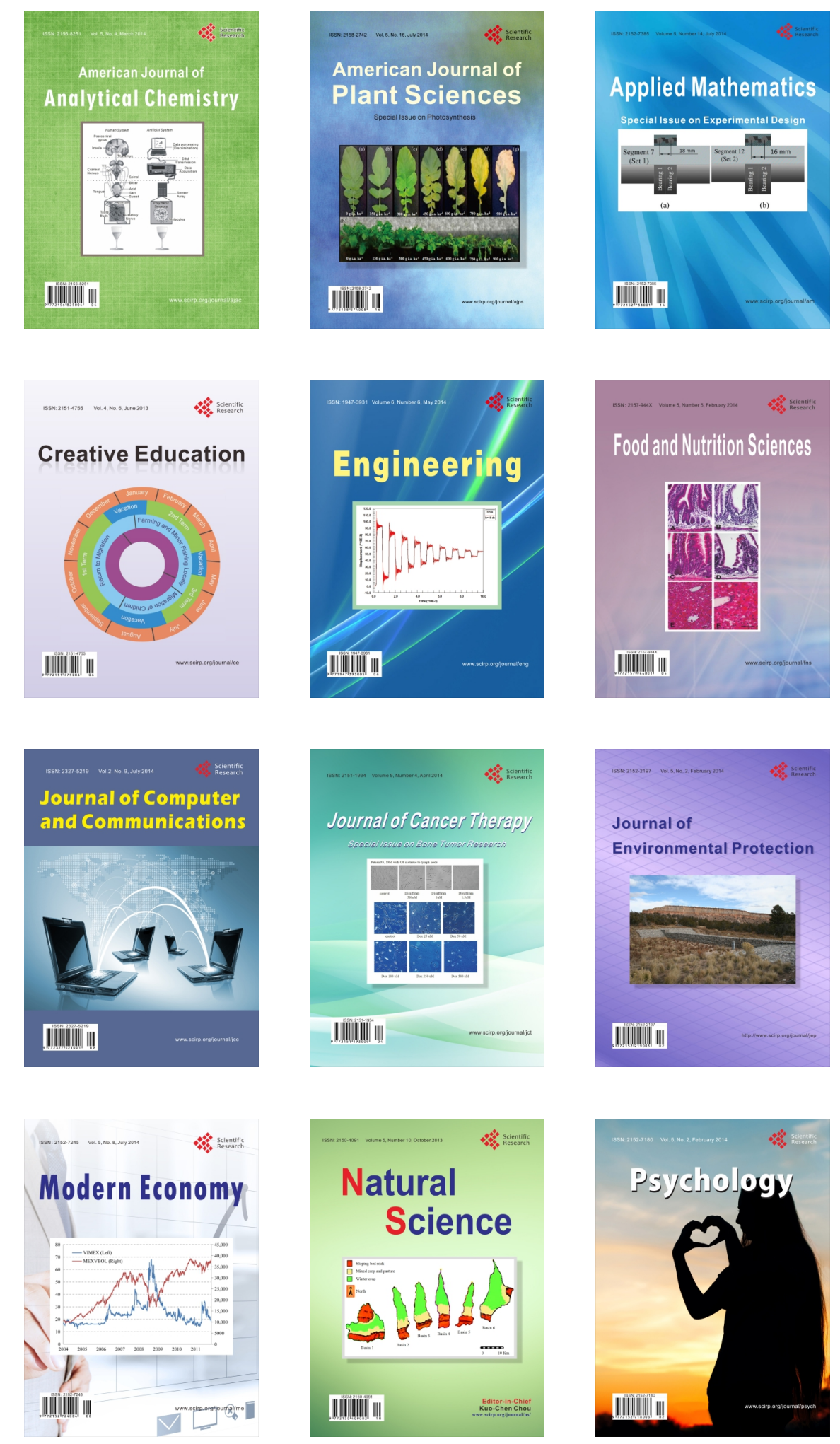